\title{
The Academic NDA: Justification, Process, and Lessons Learned
}

\author{
John J. Sunderland \\ Division of Nuclear Medicine, Department of Radiology, University of Iowa, Iowa City, Iowa
}

\begin{abstract}
The University of lowa recently completed a 4-y expedition into the uncharted waters of the Food and Drug Administration (FDA) newdrug application (NDA) process that ultimately resulted in approval of ${ }^{68} \mathrm{Ga}$-DOTATOC in August 2019. The journey was enlightening, revealing a highly structured, arcane, but rigorous regulatory approval process. The FDA proved to be an efficient, reasonable, and communicative regulatory body that achieved balance between support of the initiative and its mission-bound, process-bound duty to ensure that the application met the expected safety and efficacy standards of the agency. With several clinically valuable PET radiopharmaceuticals without intellectual property residing in regulatory limbo, without industry champions to bring them to marketing approval, there may be justification for a more concerted effort from the molecular imaging community into generating better understanding, support, and perhaps even infrastructure for the academic NDA. As a first step, this article briefly describes the start-to-finish story for ${ }^{68} \mathrm{Ga}$-DOTATOC, including a description of the clinical trials, a broad overview of the structured content of the NDA document, and the distilled experiences associated with the ${ }^{68} \mathrm{Ga}$-DOTATOC NDA process. It is anticipated that with sustained free sharing of information relating to the FDA drug registration process, it will prove less daunting and more efficient in future academically sponsored NDA filings for PET imaging agents.
\end{abstract}

Key Words: NDA; ${ }^{68} \mathrm{Ga}-\mathrm{DOTATOC}$; neuroendocrine tumor

J Nucl Med 2020; 61:480-487

DOI: 10.2967/jnumed.119.238287

$\mathbf{O}$ the morning of August 21, 2019, the University of Iowa's PET Imaging Center received official notification from the Food and Drug Administration (FDA) that the center's new-drug application (NDA) for ${ }^{68} \mathrm{Ga}$-DOTATOC injection was approved for use with PET for localization of somatostatin receptor-positive neuroendocrine tumors (NETs) in adult and pediatric patients. This ended a 4-y effort by a small team at the University of Iowa to navigate the regulatory gauntlet that is the FDA's highly structured and rigorous process for drug approval. ${ }^{68} \mathrm{Ga}$-DOTATOC is the second PET radiopharmaceutical submitted and approved by an academic institution. The Mayo Clinic received approval for ${ }^{11} \mathrm{C}$-choline injection in September 2012. In both cases, the academic institutions waived exclusivity and opened the market to both academia and industry to submit abbreviated NDAs to facilitate access of these drugs to patients.

Received Nov. 11, 2019; revision accepted Feb. 3, 2020.

For correspondence or reprints contact: John J. Sunderland, University of lowa Hospitals and Clinics, 200 Hawkins Dr., lowa City, IA 52242.

E-mail: john-sunderland@uiowa.edu

Published online Feb. 7, 2020.

COPYRIGHT (C 2020 by the Society of Nuclear Medicine and Molecular Imaging.
The road to the ${ }^{68} \mathrm{Ga}$-DOTATOC NDA application at the University of Iowa was born nearly 2 decades ago through clinical research for NET disease being performed by M. Sue O'Dorisio, Thomas O'Dorisio, and David Bushnell, who were among the first in the United States to perform ${ }^{90} \mathrm{Y}$ peptide receptor radiation therapy. The university's ${ }^{68} \mathrm{Ga}$-DOTATOC imaging program began in 2011 with the purchase of its first ${ }^{68} \mathrm{Ge} /{ }^{18} \mathrm{Ga}$ generator and the commencement of its first imaging clinical trial, "Safety of ${ }^{68} \mathrm{Ga}$-DOTA-tyr ${ }^{3}$-Octreotide PET in Diagnosis of Solid Tumors (GA-68)" in 2012. This trial was the first of 3 registered clinical trials at the University of Iowa that formed the foundation of the clinical section of the NDA.

The NDA process is not for the faint of heart. For the University of Iowa, it was a consuming 4-y journey. Although NDA submissions are fully electronic, the ${ }^{68} \mathrm{Ga}$-DOTATOC submission was over 1,300 pages and included over 200 hyperlinked documents. This is a remarkably short application by NDA standards, with its brevity attributable to the substantial use of the literature for nonclinical sections (animal study and toxicity data) and orphan drug-specific rules that allowed for fewer subjects in the pivotal trials that supported the application. For perspective, this is 10-100 times shorter than a typical therapeutic drug application for a nonorphan indication. For an academic institution, the planning, drafting, assembly, and electronic submission of the NDA to meet formatting requirements requires contracting with consulting firms. The cost of the drafting and submission process will likely run into the hundreds of thousands of dollars.

For the University of Iowa, total out-of-pocket expenses approached $\$ 400,000$, with most associated with consultant fees. Included in this total are cash expenses for team travel to the FDA. Not included are time and effort spent by the University of Iowa team. A $\$ 300,000$ grant from the Margie and Robert E. Petersen Foundation helped subsidize some personnel effort and approximately $\$ 200,000$ of the consultant fees. The core team included the author, a regulatory specialist, a statistician, a PET clinical manager (CNMT), a radiochemist, 3 nuclear medicine physicians, and a radiologist. Additional important contributions came from technologists and Holden Comprehensive Cancer Center staff. The NDA text was primarily written by the author, the radiochemist, the statistician, and our consultants. It is unclear whether these costs are typical for an academic NDA. Each situation is different, and a careful pro forma should be created to ensure that the venture makes financial sense.

The other active participant in the NDA process is the FDA itself and, more specifically, the Center for Drug Evaluation and Research and the Division of Medical Imaging Products. Our experience with the FDA throughout the entirety of the process was nothing but positive, from our face-to-face pre-investigational new-drug (IND) meeting in 2015 on through preapproval inspection and label negotiations. It is clear from our interactions that FDA personnel and leadership recognize the value of radiopharmaceuticals 
and that there appears to be an ingrained culture targeting education, facilitation, and advising in the process of NDA strategy and design. However, the role of the FDA is necessarily formal, and it remained appropriately uncompromising in its standards, throughout.

\section{JUSTIFICATION FOR NDA}

Coincidently, just 1 wk before the ${ }^{68} \mathrm{Ga}$-DOTATOC approval, the New England Journal of Medicine published a Perspective article written by independent scientists and physicians entitled "Sustainable Discovery and Development of Antibiotics: Is a Nonprofit Approach the Future?" (1). The concept for the article was driven by the recent NDA approval of pretomanid, an antibiotic used to combat a drug-resistant form of tuberculosis. The article was written by staff from TB Alliance, a not-for-profit organization whose mission includes development of affordable tuberculosis drugs. In this article, the authors propose a nonprofit discovery-and-development model for antibiotics because industry development is at a standstill due to lack of profitability.

Although not identical (there appears to currently be a healthy pipeline of new PET radiopharmaceuticals), there is a similar situation in PET in which potentially clinically valuable radiopharmaceuticals with a history of successful use in scientific studies and early-phase trials languish in regulatory limbo because they have no champion to carry them across the finish line to marketing approval. Industry champions are unlikely to surface because of limited profitability associated with drugs with no clear intellectual property or patent to be claimed. Academic champions are unlikely because academia generally lacks the necessary domain knowledge, professional motivation, and funds necessary to navigate the NDA process, which is neither easy nor inexpensive.

That said, there are compelling reasons for academic institutions to rise to the challenge of the FDA drug registration process-reasons such as benefits to the patient population, expansion of molecular imaging, and institutional economic benefit. To the academician, the economic benefit may sound shallow, but a sound business model is the most compelling component in the institutional discussion about whether to invest in the process.

\section{Sustainability}

For ${ }^{68} \mathrm{Ga}$-DOTATOC and the University of Iowa, the rationale to move forward with the NDA was severalfold, but long-term financial sustainability of our neuroendocrine imaging program was paramount. The price of newly approved radiopharmaceuticals is

\section{NOTEWORTHY}

- The Division of Medical Imaging Products at the FDA, operating within a highly restrictive environment, has demonstrated itself to be a responsive mission-driven organization working hard to empower the academic molecular imaging community to navigate PET drugs through the NDA process.

- A better understanding of the NDA structure, content, process, and level of evidentiary expectation by the molecular imaging community is an important component for downstream approval of additional PET radiopharmaceuticals.

- The NDA process is onerous but will be made more efficient through the open sharing of experiences and resources among stakeholders in the molecular imaging community that have submitted NDAs. understandably high. Companies must recoup investment from expenses from the clinical trials, the drug application process, setting up of production and distribution systems, and building of administrative infrastructure to support the enterprise. However, for imaging establishments, reimbursement is uncertain. Even with Centers for Medicare and Medicaid Services pass-through reimbursement, the University of Iowa runs only at close to break-even status imaging newer PET radiopharmaceuticals. With the recent pass-through expiration for NETSPOT (Advanced Accelerator Applications) and its associated reimbursement decrease, sizeable net losses were anticipated that will be exacerbated by nearby private hospitals ceasing to provide NETSPOT imaging for this now under-reimbursed procedure. The University of Iowa can produce ${ }^{68} \mathrm{Ga}$-DOTATOC at a fraction of the NETSPOT price and will likely be able to maintain service to the NET patient population indefinitely without financial loss.

\section{Financial Opportunity}

If an academic institution's primary motivation includes entrepreneurial pursuits, capitalizing an approved PET radiopharmaceutical through exclusive and nonexclusive licensing agreements to other institutions or industry is a possibility. This has not yet been a motivation for academic institutions bringing PET radiopharmaceuticals to approval, but it does present a revenue opportunity to channel funds into the institution's research program and help recoup the cost associated with the NDA submission.

\section{Patient Access}

At its heart and in its inception, the decision to move forward with the ${ }^{68} \mathrm{Ga}$-DOTATOC NDA was about patient access. The clinician-scientists (M. Sue O'Dorisio and Tom O'Dorisio) primarily responsible for the clinical trials that supported the NDA were passionately driven by the need to help make these state-ofthe-art PET radiopharmaceuticals available in the United States.

\section{Data Collection Standardization}

Data collection standardization strategies for nonproprietary PET drugs for clinical trials have only recently been contemplated and attempted. This attempt was largely in response to the FDA's suggestion that the academic PET community might more efficiently compile imaging and safety data from promising nonproprietary PET radiopharmaceuticals if clinical trial data were accumulated from multiple institutions using standardized methodologies. In this proposed scenario, each site could submit its own IND, with its own protocol and specific aims. However, critical aspects of the trial would be performed using standardized methodologies such that efficacy and safety data might be meaningfully combined in a downstream drug-registration (NDA) submission. Examples of clinical trial criteria that might be standardized are listed in Supplemental Table 1 (supplemental materials are available at http://jnm.snmjournals.org).

Standardization of methodologies was a critical issue that needed to be addressed in the University of Iowa ${ }^{68} \mathrm{Ga}$-DOTATOC NDA submission because it was based on 3 nonstandardized single-institution clinical trials performed over a period of $6 \mathrm{y}$. Even with these trials being performed at the same institution, all methods were not prospectively standardized. To remedy this situation, a retrospective reanalysis of the data from the 3 clinical trials was performed using standardized criteria. This process is simpler than the multiinstitutional approach.

For example, end-product specifications for ${ }^{68} \mathrm{Ga}$-DOTATOC were defined in the IND for each protocol and were identical across all 3 trials, obviating the need for standardization. For ${ }^{68} \mathrm{Ga}-$ DOTATOC, the end-product specifications were fairly simple and 
included appearance (colorless and free of particulate matter), radiochemical identity as defined by instant thin-layer chromatography and high-performance liquid chromatography (against a cold galliumDOTATOC reference standard), radiochemical purity by instant thin-layer chromatography and high-performance liquid chromatography (both $>90 \%{ }^{68} \mathrm{Ga}$-DOTATOC), and $\mathrm{pH}$ (between 4.0 and 8.0). In the context of a multicenter trial, it would be sufficient to mandate that all ${ }^{68} \mathrm{Ga}$-DOTATOC doses synthesized at all sites meet these standards.

With regard to safety, a common standard for adverse event reporting is critical so that data can be combined and properly interpreted by the FDA. Within the ${ }^{68} \mathrm{Ga}$-DOTATOC trials, adverse events were reported using standard Common Terminology Criteria for Adverse Events (version 4.0). If data were obtained from a different version of the criteria, the data were converted to version 4.0 for consistency. Adverse event information included toxicity category provided by the OnCore (Forte Research Systems) system. This information included the toxicity of the adverse event, its grade, its attribution to ${ }^{68} \mathrm{Ga}$-DOTATOC, and a description of the toxicity. Any clinical follow-up information, including the date of clinical follow-up, was collected from the subject's chart.

The dose ranges for the 3 prospective clinical trials were not identical. However, retrospectively defining a range of 111-185 $\mathrm{MBq}(3-5 \mathrm{mCi})$ included virtually all subjects in all 3 trials. A 148-MBq (4-mCi) dose was common to all trials and was ultimately the dose accepted for the label. Within a prospective multicenter trial context, defining a common dose range is critical.

Acquisition parameters should also be predefined, to the extent it makes sense. Specific to the ${ }^{68} \mathrm{Ga}$-DOTATOC trials, the uptake time targeted $60 \mathrm{~min}$ throughout the 3 prospective trials. Because uptake levels of ${ }^{68} \mathrm{Ga}$-DOTATOC are not static over time, the imaging time point is a relevant variable that requires standardization to meaningfully combine data in either a single-center or a multicenter setting.

Image interpretation and analysis in the prospective ${ }^{68} \mathrm{Ga}$ DOTATOC clinical trials differed substantially, because the aims of each of these trials were quite different. For the purposes of the NDA, in the retrospective analysis of the prospective data all ${ }^{68} \mathrm{Ga}-$ DOTATOC studies were reread independently by 2 blinded physicians. Disease positivity was assigned by each physician, and sites of disease (organ and region) were tabulated according to a protocol specifically defining rules for positivity. Disagreements between physicians were resolved by a third, independent, physician, according to the protocol. Within the context of a multicenter trial, having common reading and reporting criteria is critical to preserving the ability to combine data. Centralized reading in the multicenter setting is a common approach to ensure more standard reading of data from multicenter trials. Centralization is possible even in the academic setting if images are uploaded to a common, secure PACS.

Lastly, the definitions of the reference standard for disease positivity and negativity for the 3 prospective ${ }^{68} \mathrm{Ga}$-DOTATOC trials were not consistent. In the retrospective analysis, we developed a standardized approach. Overall, a combination of pathology, OctreoScan imaging, conventional imaging, and biomarker data results was used to determine whether a subject was positive or negative for NET disease. The positive-for-NET-disease classifications were further delineated into types $1-4$ on the basis of the strength of the data indicating disease status. By design, type 1 evidence of NET disease (pathology) was considered more accurate for determination and superseded type 2 evidence (OctreoScan or ${ }^{68} \mathrm{Ga}$-DOTATATE). Type 2 was considered less accurate than type 1 but more accurate than and superseding type 3 (multiple sites of disease on conventional imaging). Type 3 was considered less accurate than either type 1 or type 2 but more reliable than and superseding type 4 (single site of disease on conventional imaging coupled with abnormally high biomarkers). The flowchart in Supplemental Figure 1 shows how this further classification was determined. In the context of prospective multicenter trials, consistent definitions for tissue positivity and negativity should be determined by a common standard or algorithm to allow for downstream combining of data.

\section{THE NDA PROCESS FOR ${ }^{68}$ GA-DOTATOC}

The University of Iowa began performing ${ }^{68} \mathrm{Ga}$-DOTATOC scans in clinical trials in 2012. Ultimately, 3 phase 2 clinical trials were initiated. The first was "Safety \& Efficacy of ${ }^{68} \mathrm{Ga}$-DOTA-tyr3Octreotide PET/CT in Diagnosis, Staging \& Measurement of Response to Treatment in Patients With Somatostatin Receptor Positive Tumors: Comparison to Octreoscan Plus High-Resolution, Contrast Enhanced CT" (NCT01619865). The second was "Comparator Study of ${ }^{68} \mathrm{Ga}$-DOTATOC PET/CT with OctreoScan + High-Resolution, Contrast-Enhanced CT for Diagnosis and Staging in NETs and Other Somatostatin Receptor Positive Tumors" (NCT01869725). The third was "Impact of Ga-68 DOTATOC PET-CT Imaging in Management of Neuroendocrine Tumors" (NCT02441062).

The data collected on the research subjects appropriately targeted the scientific aims of the clinical trials and were not initially collected with the intention of being used in a regulatory filing. As such, only single physician reads were performed, and definitions of the reference standard for disease changed from trial to trial. Data collected from each trial were understandably different. For example, in the Change in Clinical Management trial, the results of follow-up conventional imaging were not necessarily collected as part of the protocol, but they proved necessary for our ultimate definition of the reference standard for tissue positivity for the NDA.

In 2015, when the research team decided that moving forward with a ${ }^{68} \mathrm{Ga}$-DOTATOC NDA was both realistic and an important institutional goal, we needed to develop a strategy to meet regulatory requirements. Our first step was to achieve orphan drug status for ${ }^{68} \mathrm{Ga}$-DOTATOC through transfer of the orphan drug designation from the Society of Nuclear Medicine and Molecular Imaging (original holder) to the University of Iowa.

The entirety of the NDA process took more than $4 \mathrm{y}$ to complete, beginning with our pre-IND meeting on April 30, 2015 (Fig. 1), moving through our pre-NDA meeting in the fall of 2017, and ending with the final approval letter issued on August 21, 2019 (Fig. 2). The submission of an NDA is the culmination of several steps, some sequential, others performed in parallel. The major steps in the NDA process are outlined in Table 1 and are described in more detail in the following sections.

\section{Step 1: Pre-IND Meeting with FDA}

There are 2 kinds of NDA application described in FDA regulations. The first and most common is the 505(b)(1) application. This is the standard prospective phase 3 trial approach in which the application contains the full reports of the multicenter clinical trials (usually 2) of safety and efficacy, nonclinical development (animal and in vitro studies), and chemistry, manufacturing, and control (CMC) information. The second type is the 505(b)(2) application, which is for studies not conducted by or for the applicant, and for which the applicant has not obtained a right of reference. This type of submission relies on information in the public domain to fulfill some of the information required in the NDA application. For ${ }^{68} \mathrm{Ga}-$ DOTATOC, the 505(b)(2) pathway was the appropriate approach. 


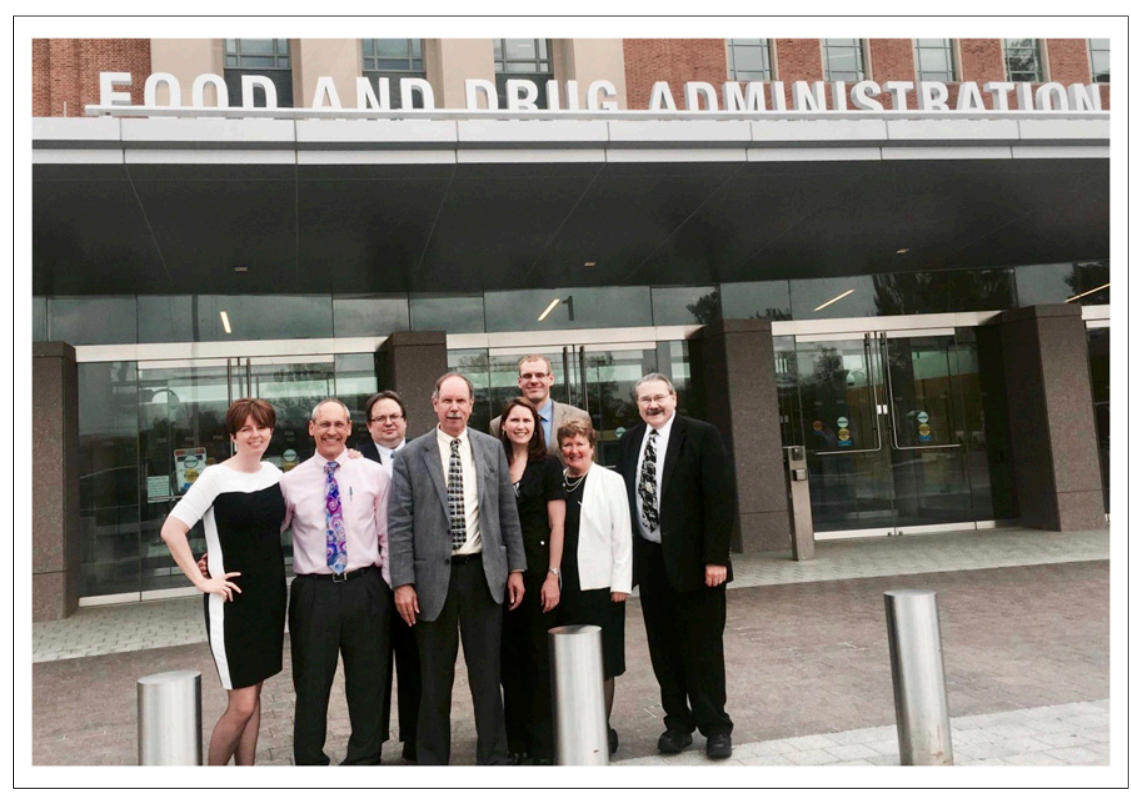

FIGURE 1. Pre-IND meeting of University of lowa ${ }^{68} \mathrm{Ga}$-DOTATOC clinical, regulatory, and scientific team at FDA on April 30, 2015. From left to right are shown Kellie Bodeker, John Sunderland, Yusuf Menda, Michael Graham, Shannon Lehman, David Dick, M. Sue O'Dorisio, and Tom O'Dorisio.

importantly, it contained a list of specific questions to the FDA on the strategy for the structure, performance, and content of the clinical trials proposed to support the presumed NDA filing.

The FDA's written response and meeting, which took place on the FDA campus on April 30, 2015, was highly productive, with the FDA providing substantive guidance on a metaanalysis and literature review, as well as approaches to making our ongoing clinical trials of sufficient rigor to meet the expected standards. Additional information was provided to help navigate the nonclinical and clinical pharmacology sections of the NDA.

\section{Step 2: Clinical Trial Planning}

On the basis of the input from the FDA and with the aid of external consultants, the University of Iowa created a new phase 3 retrospective clinical trial, which consisted of a rigorous retrospective analysis of the combined subjects enrolled in the original 3 prospective trials. The single retrospective trial was designed to harmonize the data

Typically, NDAs require 2 independent, large multicenter trials to provide data to support the clinical claim of the regulatory filing. Neuroendocrine cancer is an orphan disease with a limited patient population, making collection of data from sufficient numbers of patients challenging. This is a recognized regulatory dilemma, and so requirements are relaxed to a minimum in the case of a drug for an orphan disease. The FDA has some limited latitude to determine the level of evidence required to inform the benefit-risk assessment that underpins orphan drug product approval. This latitude largely means that fewer patients are required in the supporting trials; it does not mean a lessening of rigor in other areas.

In February 2015, the University of Iowa submitted a pre-IND meeting request and briefing package to the FDA with the expressed purpose of reviewing the extensive published data and the data generated from University of Iowa clinical trials of ${ }^{68} \mathrm{Ga}$-DOTATOC to assess adequacy for filing a 501(b)(2) NDA. The briefing package is a formal document with a mandated format whose content is sufficient to inform the FDA of the salient information about the drug, including its route of administration, its proposed indication, a summary of the peer-reviewed literature, and, in our case, an update on the current status of our ongoing ${ }^{68} \mathrm{Ga}$-DOTATOC trials. Most collected, the reference standard definitions, and the analysis approach. In some cases, additional clinical data were gathered that were not collected in the initial trials. A completely new clinical trial protocol was written describing in detail the inclusion criteria, the necessary imaging and clinical data collected, the reference standards, the primary and secondary endpoints, the statistical endpoints, and the statistical analysis plan (SAP).

In the new protocol, patient studies were reread in a blinded manner with standardized case report forms by at least 2 qualified physicians. Additional clinical data, imaging data, analysis, reference standards for disease positivity and negativity, and safety data were similarly harmonized into a single approach. Under these conditions, some patients from the initial trials were necessarily excluded from the final analysis.

In parallel with the clinical trials, the University of Iowa authored a protocol for a formal metaanalysis of the literature in collaboration with one of the university's resident librarians, who is a metaanalysis expert. This effort incorporated FDA suggestions on the design of the metaanalysis protocol to enhance the probability that it would meet the FDA's scientific evidentiary expectations. Statistical endpoints for sensitivity and specificity were predefined.

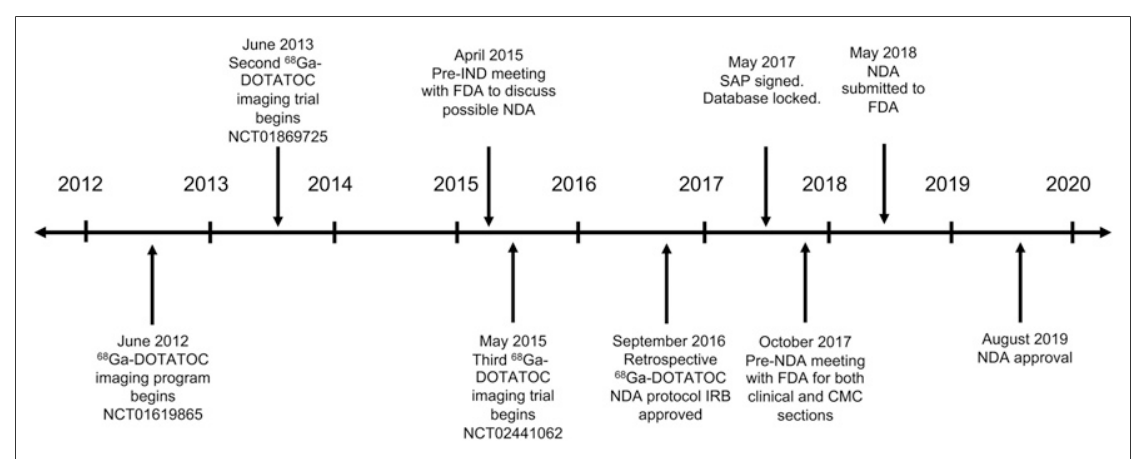

FIGURE 2. Timeline for ${ }^{68} \mathrm{Ga}-\mathrm{DOTATOC}$ NDA project. IRB $=$ institutional review board.

\section{Step 3: Performance of Clinical Trial}

The performance of the retrospective clinical trial, including collection and documentation of all data, generation of completed case report forms, blinded rereads of ${ }^{68} \mathrm{Ga}$-DOTATOC studies, and rereading of conventional imaging scans (CT, MRI, ${ }^{111}$ In-OctreoScan) for the more than 350 subjects, took over a year to complete.

In parallel, the formal ${ }^{68} \mathrm{Ga}$-DOTATOC metaanalysis was performed and ultimately published in The Journal of Nuclear Medicine (2). Efficiencies were achieved in the writing of sections of the NDA by having 


\begin{tabular}{|c|c|c|}
\hline Activity & Description & Comment \\
\hline $\begin{array}{l}\text { 1. Pre-IND meeting } \\
\text { with FDA }\end{array}$ & $\begin{array}{l}\text { Proposed clinical trial and overall NDA } \\
\text { approach are discussed, with opportunity to } \\
\text { ask questions about FDA's opinion on any } \\
\text { aspect of trial or NDA strategy. }\end{array}$ & $\begin{array}{l}\text { Meeting request is lengthy formal document } \\
\text { with background material sufficient to } \\
\text { familiarize FDA with product and situation. } \\
\text { The only topics discussed are those related } \\
\text { to questions asked in package. }\end{array}$ \\
\hline $\begin{array}{l}\text { 2. Clinical trial } \\
\text { planning }\end{array}$ & $\begin{array}{l}\text { Protocols are written with aims designed to } \\
\text { support proposed label indication. Statistics } \\
\text { are critical. Reference standards must be } \\
\text { clearly defined. Data collection must be } \\
\text { designed to withstand FDA audit. }\end{array}$ & $\begin{array}{l}\text { SAP is formal, signed, document and must be } \\
\text { part of formal clinical trial. Analysis approach } \\
\text { and statistical endpoints for proposed } \\
\text { indication are committed a priori. }\end{array}$ \\
\hline $\begin{array}{l}\text { 3. Performance of } \\
\text { clinical trial }\end{array}$ & $\begin{array}{l}\text { Trial data are collected and formally } \\
\text { documented. }\end{array}$ & $\begin{array}{l}\text { Internal audits as trial progresses are important } \\
\text { to ensure data are collected completely and } \\
\text { correctly. }\end{array}$ \\
\hline $\begin{array}{l}\text { 4. Data lock and } \\
\text { statistical } \\
\text { analysis }\end{array}$ & $\begin{array}{l}\text { All trial data are entered into FDA prescribed } \\
\text { data format for statistical analysis. Database } \\
\text { is locked. Statistical analysis is performed } \\
\text { precisely according to SAP. }\end{array}$ & $\begin{array}{l}\text { Before data lock, all trial data must be reviewed } \\
\text { for accuracy and completeness. Statistical } \\
\text { analysis is performed using SAS software } \\
\text { versions prescribed by FDA. SAS code is } \\
\text { submitted in application. }\end{array}$ \\
\hline $\begin{array}{l}\text { 5. Pre-NDA meeting } \\
\text { with FDA }\end{array}$ & $\begin{array}{l}\text { Preliminary safety and efficacy analysis results } \\
\text { are shared with FDA. Questions to FDA about } \\
\text { adequacy of data and questions on structure } \\
\text { and content of NDA are discussed. }\end{array}$ & $\begin{array}{l}\text { From output of this meeting, likelihood that } \\
\text { data are sufficient to support indication } \\
\text { should be clear. All major questions about } \\
\text { format and content of application should } \\
\text { have been answered. }\end{array}$ \\
\hline $\begin{array}{l}\text { 6. Writing of NDA } \\
\text { document }\end{array}$ & $\begin{array}{l}\text { NDA must be written and formatted into eCTD } \\
\text { format, which consists of } 5 \text { modules. Module } \\
5 \text { is clinical trial study reports, clinical trial } \\
\text { information, and tabulated data. Module } 4 \\
\text { is nonclinical animal data. Module } 3 \text { is } \\
\text { chemistry manufacturing and controls. } \\
\text { Module } 2 \text { is introduction and summaries of } \\
\text { modules } 3-5 \text {. Module } 1 \text { is label and other } \\
\text { institutional information. }\end{array}$ & $\begin{array}{l}\text { eCTD submissions for NDAs have been } \\
\text { required since } 2017 \text {. Data within eCTD } \\
\text { document have additional FDA-required } \\
\text { formats, including clinical datasets, statistical } \\
\text { code, and label formatting. These standards } \\
\text { are changing. Implications for academic } \\
\text { institutions submitting NDAs are unclear, but } \\
\text { more expense will likely be required. }\end{array}$ \\
\hline 7. Submission & $\begin{array}{l}\text { Submission is via electronic eCTD submission. } \\
\text { Electronic receipt will be issued when it } \\
\text { passes FDA gateway. }\end{array}$ & \\
\hline $\begin{array}{l}\text { 8. Review and } \\
\text { request for } \\
\text { information }\end{array}$ & $\begin{array}{l}\text { FDA has } 2 \text { mo to review application for } \\
\text { completeness. FDA can refuse regulatory } \\
\text { filing if critical information is missing or is of } \\
\text { insufficient quality. If accepted, FDA has } \\
\text { additional } 6 \text { mo for priority review or } 10 \text { mo } \\
\text { for standard review. FDA will file additional } \\
\text { requests for information during review. }\end{array}$ & $\begin{array}{l}\text { Thirteen requests for information were } \\
\text { received. The most serious addressed an } \\
\text { algorithmic error in reference standard } \\
\text { determination impacting classification of } \\
\text { a handful of subjects. Delay in correcting } \\
\text { and resubmitting tabulated data delayed } \\
\text { Prescription Drug User Fee Act date by } 3 \text { mo. }\end{array}$ \\
\hline
\end{tabular}

the metaanalysis published in the peer-reviewed literature before the NDA submission.

\section{Step 4: Data Lock and Statistical Analysis}

The NDA is a highly formalized, high-stakes process. To maximize the rigor and transparency of the clinical trial process, rigid controls are used. For the purposes of an NDA, data are meant to be collected precisely according to the written protocol. In this way, the FDA is in a position to know what data are available for inspection or audit. Further, the data must be entered into an FDAmandated database format. The SAP must be completely and formally prespecified. The statistical analysis must be performed using a specified SAS software version such that the FDA can duplicate or perform its own analyses. Both the SAS analysis code and the tabulated data are submitted as part of the NDA.

On May 5, 2017, the University of Iowa completed, signed, and dated the final version of the SAP. After a completed internal audit of the clinical trial data, the database was locked on May 15, 2017. A preliminary global analysis of the safety and efficacy data was performed using the statistical analysis described in our SAP. A preliminary assessment of sensitivity and specificity was completed, and we compiled our overall safety data. Within 2 mo of our database lock, the preliminary analyses uncovered 2 small errors in our clinical data. Twice, we unlocked the database to correct these errors. Unlocking of the database and the associated changes were fully disclosed in the NDA. 


\section{Step 5: Pre-NDA Meeting with FDA}

After completion of data collection and the analysis of our retrospective phase 3 trial-and completion (but not yet publication) of the metaanalysis - a formal pre-NDA meeting was scheduled with the FDA. The explicit purpose of the requested meeting was to agree on the content and format of a complete 505(b)(2) application for marketing approval of ${ }^{68} \mathrm{Ga}$-DOTATOC. At the FDA's request, 2 separate meetings were scheduled, the first to discuss the clinical and nonclinical aspects, which occurred on October 3,2017 , and the second to allow for CMC questions, which occurred on October 10, 2017.

The pre-NDA briefing package was prepared with the help of external consultants familiar with PET radiopharmaceutical NDAs. In the pre-NDA package for the clinical portion of the NDA, summary results of our retrospective trial and metaanalysis were presented. The FDA was asked to judge the initial perceived adequacy of our clinical evidence of safety and efficacy and was explicitly asked whether additional evidence would be required.

Most subsequent questions were related to strategies associated with providing necessary information in the NDA with format and content acceptable to the FDA. The primary purpose of these questions was to explore acceptable efficiencies in the presentation of evidence within the constraints of the NDA structure.

\section{Step 6: Writing the NDA Document}

The NDA is a highly formal document both in content and in structure and must be submitted as an electronic common technical document (eCTD). eCTD is a standard interface and international specification for pharma to transfer regulatory information to the FDA (or another international agency). It is based on the commontechnical-document format that was developed by the International Council for Harmonization. It is important to understand that the eCTD format is designed not for the convenience of the submitting organization but to help the FDA efficiently review the NDA. Writing, organization, and compilation of the NDA into the eCTD structure, with its hierarchical headings and subheadings, is a tedious

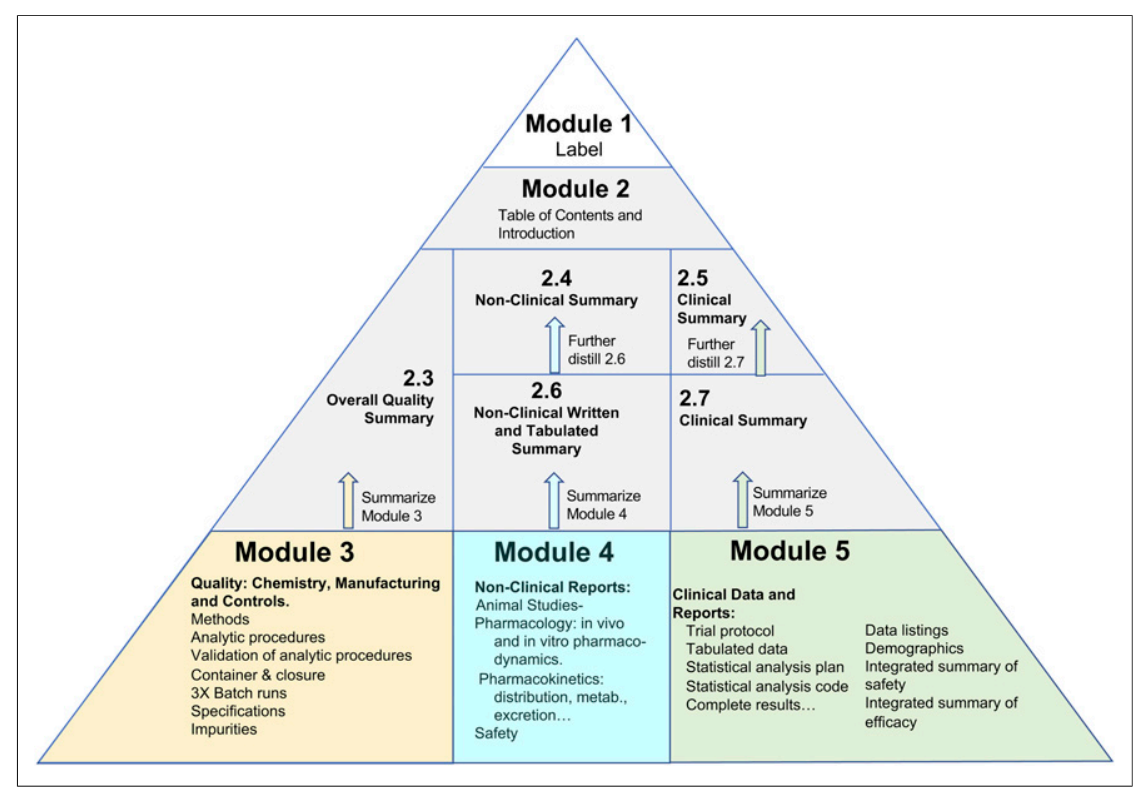

FIGURE 3. Pyramid representation of eCTD format required for use in NDA drug registration submissions, annotated with module contents. and time-consuming endeavor and requires an external consultant to assemble and submit.

The NDA itself, as illustrated in Figure 3, consists of 5 modules. The application is largely drafted from bottom to top, starting with module 3 , the quality/chemistry section; module 4, nonclinical data; and module 5, the clinical trial data and the trial reports. Each of these sections is independent of the others, and the sections can be drafted in parallel.

Module 3, the quality/chemistry section, is where CMC information is detailed. The level of detail expected in a marketing approval application is far in excess of what is typically required for an IND drug. Drug master files from vendors for synthesis modules or cassettes are helpful in simplifying the application. Manufacturers of synthesis modules, cassettes, or even chemical precursors are not only subject to FDA inspection but also likely to be inspected by the FDA if they have not been previously inspected; this includes overseas manufacturers. The applicant's site will absolutely be inspected by the FDA as part of the preapproval inspection process. The inspection will be rigorous, and the site will be responsible for adherence to procedures and information precisely as described in module 3 .

Module 4 reports the nonclinical (animal) data that support the application. Because all animal studies for ${ }^{68} \mathrm{Ga}$-DOTATOC came from the literature (as was agreed to by the FDA in the pre-NDA meeting), the University of Iowa application simply included electronic (pdf) versions of all the original articles that contained the pharmacodynamic, pharmacokinetic, and toxicology data necessary to support the application. All salient article data and results were summarized in the appropriate subsections in module 2, section 2.6.

Module 5, including the clinical study reports, was the most extensive section in the ${ }^{68} \mathrm{Ga}$-DOTAOC NDA. It contained not only the clinical trial reports but also the clinical trial protocols, SAP, case report forms, informed consent forms, institutional review board approval documents, and listing of study team members. Module 5 also contained the full tabulated data for all research subjects. The body of the clinical study report was the longest single document in our NDA, describing the clinical trial objectives, the detailed investigative plan, all study variables, the safety measures, the quality assurance methods, the statistical methods, and the methods for determining the sample size. It also summarized the trial results, including efficacy evaluations (sensitivity, specificity) and safety evaluations, each broken down by subgroups (i.e., race, age, and sex).

Module 2 presents high-level summaries of the results of modules 3-5 and can be written only after these modules are functionally complete. Critical data, results, and summaries from modules 3-5 are used to populate the summaries in module 2 . The clinical summary in section 2.7 distills the clinical study report included from module 5. For perspective, this "summary" was approximately 100 pages in length, which is brief, considering the FDA's 400 page limit for this section. Section 2.6, the nonclinical summary, totaled approximately 60 pages, which for our application was a summary of available pharmacokinetic, pharmacology, and toxicity literature for ${ }^{68} \mathrm{Ga}$-DOTATOC, with relevant information 
summarized in the appropriate subsections. One required component of module 4 is data on repeat-exposure dose toxicity, for which no ${ }^{68} \mathrm{Ga}$-DOTATOC data existed in the literature. Fortunately, Isotopen Technologien München AG, a pharmaceutical company with interest in commercializing DOTATOC radiopharmaceuticals, identified the same void and contracted a third party to perform the necessary repeat-dose toxicity study for DOTATOC on rats. The company graciously provided a letter of reference to these data in support of the ${ }^{68} \mathrm{Ga}$-DOTATOC NDA.

Section 2.3 , the quality/CMC summary for ${ }^{68} \mathrm{Ga}$-DOTATOC, consisted of 3 sections summarizing the quality of the active drug product (the vial of ${ }^{68} \mathrm{Ga}$-DOTATOC) and the overall summary of the precursor (cold DOTATOC) and the drug substance (the ${ }^{68} \mathrm{Ga}-$ DOTATOC drug itself). The quality overall summary was brief, as it largely outlined only the molecular structure, ${ }^{68} \mathrm{Ga}$ decay scheme and emissions, and safety and decay information. The section on the quality of the active drug product was a more extensive document including manufacturing processes, process control, control of excipients and drug product, reference standards, container and closure systems, and stability data.

The nonclinical and clinical overviews (sections 2.4 and 2.5) are written as a very brief high-level description of the data supporting the application and were approximately 10 and 20 pages, respectively. These documents refer liberally to the actual application data from modules 4 and 5 supporting the proposed indication and clinical use.

Lastly, module 1 allows for geographically specific and sitespecific information to be presented and, importantly, also contains the label. The FDA places special emphasis on the label, the significance of which was underappreciated until we proceeded through the NDA authoring process. The label is the quintessence of the NDA and is the final distillation of the entire application into a single summary document. What we did not know at the outset is that the FDA requires 2 labels to be submitted in the NDA. One is the clean label, or package insert, that we are accustomed to seeing. The second is the annotated label. The annotated label is identical to the original label except that every statement on the label contains hyperlinks to the specific section of the NDA that contains the specific data that support that particular entry.

\section{Step 7: Submission}

Submission of the NDA to the FDA is a process in itself. Each of the several hundred individual documents written for specific entries in the NDA must be loaded into eCTD-generating software. Each document contains both internal hyperlinks to navigate within the document and external hyperlinks that link to other documents elsewhere in the NDA. There are several thousand hyperlinks, all of which need to be checked for functionality before submission. The eCTD software is prohibitively expensive for most academic institutions, and so consultants were hired to complete the assembly and testing of the eCTD-formatted NDA. Once the compiled NDA was verified as complete, functioning, and accurate, submission was authorized.

\section{Step 8: Review and Request for Information}

The submission of an NDA is a momentous event. However, it is also just the beginning of the next phase: the review.

By Prescription Drug User Fee Act statute, the FDA targets a period of 2 mo to review the application for completeness. The FDA can refuse the regulatory filing if critical information is missing or the application is of insufficient quality. If the application is accepted, the FDA has an additional 6 mo for priority review or 10 mo for standard review. The FDA can, and will, file requests for additional information, which may include clarifications or additional analyses. Because the FDA is on a tight review timetable, all issued requests for information also contain an aggressive deadline by which responses must be received. If the FDA identifies problems of sufficient magnitude that delays in the review will result, it can and will extend the Prescription Drug User Fee Act date beyond the original timetable. The University of Iowa responded to 13 requests for information, in total. The most frequent requests were related to the quality/CMC section. The most serious request was related to a small algorithmic error in our determination of the reference standard, impacting the disease classification of a handful of subjects. The fact that this algorithmic error was discovered is a testament to the detail with which the FDA conducts its review. Fewer than 5 subjects were impacted by the error, but the delay in correcting and resubmitting the tabulated data and associated statistical analysis was enough that the FDA requested a 3-mo extension of the Prescription Drug User Fee Act date.

There are 3 benchmark events that the FDA is required to schedule during the course of the review. The first is the midcyclereview phone call, which takes place roughly halfway through the review process. During this phone call, representatives of the FDA's review subsections are present. In all, 24 FDA individuals attended this call, with representatives from the Center for Drug Evaluation and Research, Division of Medical Imaging Products, Division of New Drug Products, CMC, Division of Clinical Pharmacology, Biostatistics/Division of Biometrics, Division of Epidemiology, and Division of Microbiology. Each group was given an opportunity to present the status of its review and ask any questions. The second benchmark is an on-site preapproval inspection. In our case, this was limited to a quality/CMC inspection of our manufacturing processes. A clinical inspection of trial data is also likely. The third benchmark event is the late-cycle meeting, which occurs near the end of the review. This is another large phone-meeting attended by the same groups as in the midcycle call. Any lastminute issues are discussed during this call.

\section{Step 9: Label Negotiation}

The label is the quintessence of the NDA and is the distillation of all the data and information presented in the application. It is the single document that physicians and the public have access to regarding the safety and efficacy of the drug. The FDA has gone to great effort to format this document as an organized, concise presentation of all the critical information from the entirety from the NDA application.

The stakes associated with label language are high, as reimbursement, and therefore clinical use, are inextricably tied to wording used in the label indication. The FDA and sponsor must agree on the label indication language. Most critically, the data in the application must unequivocally support the claimed label indication.

\section{LESSONS LEARNED}

Through the entirety of the process of planning, drafting, and submitting an NDA, the learning curve was steep and continuous. Lessons were learned through interacting with the FDA, which was both patient and professional throughout; listening to our consultants, who had submitted several PET NDAs previously; reading and understanding the regulations surrounding the NDA process; listening to colleagues in academia and industry; and finally, attending educational events organized by the Society of Nuclear Medicine and Molecular Imaging and the FDA. 
One of the primary lessons learned is that what academicians consider clear and compelling evidence for clinical safety and efficacy does not match the level and detail of rigor demanded by the FDA. The FDA has a decades-long formal process for drug approval, and although there is some inherent flexibility in approach, it remains a rigid process with requirements on which the FDA is necessarily unyielding. Academia is generally unappreciative of the expected rigor.

The critical role of biostatistics in the NDA process, and the central role and workload assumed by the primary biostatistician, were surprising. The NDA is based on data. The biostatistician is the steward of that data. As such, our biostatistician was a more critical and active team member than anticipated. The statistical methods used were fundamentally mundane; however, the depth of the algorithmic development was substantial. The writing of the SAS analysis code was consuming both during the NDA data analysis process and during the review, when the FDA asked for additional subanalyses to be performed. The FDA reviews the SAS code and variable definitions carefully.

The University of Iowa has submitted many PET IND applications and is no stranger to CMC submissions. However, the amount of additional information necessary and the overall rigor and detail required in the NDA application were a revelation. Two synthesis modules were initially included in the NDA, but one had neither a drug master file submitted nor the resources to generate a drug master file or the necessary process-control data, and so it was removed from our application.

One final important lesson learned was that the quality of molecular imaging literature describing the investigation of radiopharmaceuticals is fundamentally poor by FDA standards. Peerreviewed literature about well-performed studies can be used to fulfill certain evidentiary requirements of an NDA using the 505(b)(2) pathway, thereby substantially simplifying the NDA process. However, most published studies lack either the necessary scientific rigor or the reporting of simple, yet critical, elements of their clinical trial methodology or results. For example, the literature can be used to support the safety of a PET drug if the reported study includes a simple statement of drug-related adverse events. Most do not and therefore cannot be included to support safety. Many studies do not perform blinded reads or use multiple readers for purposes of reader agreement, which is an important FDA metric. Reference standards are often weak or poorly defined. Too many studies are retrospective and not prospectively designed. Some, but not all, of these issues are related to a lack of funding to perform these trials with the necessary rigor-an understandable limitation.

The FDA has been actively encouraging a model whereby clinical data for PET radiopharmaceuticals in the public domain might be collected more efficiently for purposes of a downstream regulatory submission. This approach consists of a common foundational harmonized protocol shared among academic institutions for a given radiopharmaceutical, probably in a late phase 2 environment. Harmonized criteria might include common radiopharmaceutical end-product specifications, an identical injected-dose range and imaging time, a common set of case report forms so that collection of clinical and safety information is harmonized, and a commonly defined reference standard for disease positivity and negativity. This does not mean that all trials from all institutions must have identical patient populations or specific aims. Each may have its own study design and endpoint. But this approach will allow the more seamless combining of data into a potentially powerful multiinstitutional trial (Supplemental Fig. 1 and Supplemental Table 1). The Society of Nuclear Medicine and Molecular Imaging's Clinical Trials Network has performed this function in the past at the FDA's request. It is a potentially powerful model but difficult to implement with limited resources.

Candidate radiopharmaceuticals for additional academic NDAs for PET exist, but certain criteria must be met. The radiopharmaceutical should be in the public domain, without intellectual property attached. Ideally, rich literature should exist supporting its safety (including FDA-required toxicology studies, pharmacokinetics, and pharmacodynamics) and efficacy in prospective, well-controlled trials. The latter data are particularly problematic, and likely additional wellcontrolled multicenter studies will be required. Finally, the business model for the submitting institution in terms of cost and effort to assemble a full NDA versus downstream revenue needs to make financial sense. Examples of candidate radiopharmaceuticals include ${ }^{15} \mathrm{O}$-water (perfusion), ${ }^{18} \mathrm{~F}$-FLT (proliferation), ${ }^{18} \mathrm{~F}$-fluoromisonidazole (hypoxia), and ${ }^{18} \mathrm{~F}$-fluoroethyltyrosine (brain tumors).

\section{CONCLUSION}

Only a handful of PET radiopharmaceuticals are currently FDAapproved drugs. However, there are at least 5 promising PET drugs in late development or in the NDA review pipeline. Most are being developed by private industry, but others are in the academic or notfor-profit arena. These include ${ }^{64} \mathrm{Cu}$-DOTATATE, ${ }^{68} \mathrm{Ga}$ PSMA, ${ }^{18} \mathrm{~F}$ DCFPyL, ${ }^{18} \mathrm{~F}$-fluoroestradiol, and ${ }^{15} \mathrm{O}$-water. What is particularly interesting about this list, including the 3 most recent PET drug approvals (NETSPOT, Axumin [Blue Earth Diagnostics, Inc.], and ${ }^{68} \mathrm{Ga}$-DOTATOC), is that the NDA approaches and strategies have all been somewhat different. That is, most have not followed the traditional pathway of 2 large, controlled multicenter phase 3 trials. It appears that most are using variations of the 505(b)(2) pathway, and the FDA is being open-minded about evidentiary sources to meet the requirements of 505(b)(2). The University of Iowa benefitted substantially from knowledge gained from the previous experiences of both academic and industry organizations that have braved the NDA process. Continued sharing of both successful and unsuccessful strategies among partners in this field, combined with continued cooperation and open communication with the FDA, is a sound strategy for advancing the field of molecular imaging more rapidly.

\section{DISCLOSURE}

Financial support was received from the Margie and Robert E. Petersen Foundation. No other potential conflict of interest relevant to this article was reported.

\section{ACKNOWLEDGMENTS}

Appreciation is extended to the University of Iowa NDA team, including Sue and Tom O'Dorisio, Michael Graham, Yusuf Menda, Janet Pollard, Nadine Mallak, David Bushnell, Xiaomei Gu, Lisa Dunnwald, Mary Schall, Diane Soulek, Allie Tucker, Shannon Lehman, Kellie Bodeker, Tim Ginader, David Dick, and Facet Life Sciences.

\section{REFERENCES}

1. Nielsen TB, Brass EP, Gilbert DN, Bartlett JG, Spellberg B. Sustainable discovery and development of antibiotics: is a nonprofit approach the future? N Engl J Med. 2019:381:503-505.

2. Graham MM, Gu X, Ginader T, Breheny P, Sunderland JJ. ${ }^{68}$ Ga-DOTATOC imaging of neuroendocrine tumors: a systematic review and meta-analysis. $\mathrm{J} \mathrm{Nucl}$ Med. 2017;58:1452-1458. 